\title{
Statistical Characteristics of New Type Internal Wave in the Ionospheric F Region
}

\author{
Jandieri George ${ }^{1, ~ *}$, Rahul Kaushik ${ }^{2}$, Tugushi Nika ${ }^{3}$ \\ ${ }^{1}$ Physics Dept., Georgian Technical University, GTU, Tbilisi, Georgia \\ ${ }^{2}$ ECE Dept., Jaypee Institute of Information Technology, JIIT, Noida, India \\ ${ }^{3}$ Energetic Dept., Georgian Technical University, GTU, Tbilisi, Georgia
}

\section{Email address:}

georgejandieri7@gmail.com (J. George),rahul.kaushik@jiit.ac.in (R. Kaushik), tugushinika96@gmail.com (T. Nika)

*Corresponding author

\section{To cite this article:}

Jandieri George, Rahul Kaushik, Tugushi Nika. Statistical Characteristics of New Type Internal Wave in the Ionospheric F Region. International Journal of Astrophysics and Space Science. Vol. 5, No. 4, 2017, pp. 55-62. doi: 10.11648/j.ijass.20170504.11

Received: June 21, 2017; Accepted: July 5, 2017; Published: October 31, 2017

\begin{abstract}
Second order statistical moments of new internal MHD wave in the ionospheric F region are investigated analytically by geometrical optics approximation. Degree of a curvature of a constant phase surface and the variance of an instant frequency measuring by experiment has been obtained for arbitrary correlation function of electron density fluctuations. Energy exchange between the internal wave and turbulent plasma flow is considered calculating the mean energy flux density in the first and second approximations. Numerical calculations are carrying out for both anisotropic Gaussian correlation function and power law-spectrum characterizing elongated plasma irregularities using experimental data of satellites and incoherent scatter radar observations.
\end{abstract}

Keywords: Ionospheric Plasma, Internal Wave, Plasma Irregularities, Statistical Moments

\section{Introduction}

At present time it was established that statistical characteristics of wave depend on as the correlation features of the variable parameters of a chaotically random medium, as well as on the dispersion and the type of waves. The features of transverse electromagnetic waves and low-frequency waves in different turbulent media were investigated quite well [1-3]. However fluctuation features of the longitudinal waves and different MHD waves are not investigated in detail. Propagation of MHD waves in the turbulent plasma flow is of great interest in both cosmic and laboratory plasma.

The Earth magnetic field in the ionosphere generates small and medium-scale MHD waves: Alfven and magneto-acoustic waves. The last one are fast (propagation velocity more that 1 $\mathrm{km} / \mathrm{s}$ ) and short-period (of the order of 5-20 min) waves generating by elasticity of the geomagnetic lines of force. Alfven waves are generated due to tension of the geomagnetic lines of force and its velocity depends on the orientation of the wave vector with respect to the geomagnetic field $\mathbf{H}_{\mathbf{0}}$. They can be very slow (10-50 m/s) and long period (1-2 days) when the wave vector $\mathbf{k}$ is almost transversal to $\mathbf{H}_{\mathbf{0}}$, and fast, when vectors $\mathbf{k}$ and $\mathbf{H}_{\mathbf{0}}$ are parallel. Some peculiarities of the second order statistical moments of scattered Alfven and magneto-acoustic waves in the turbulent plasma flow were considered in [4-6].

We suppose that the external magnetic field $\mathbf{H}_{\mathbf{0}}$ is directed along the $\mathrm{z}$-axis and turbulent plasma flow propagates with the mean constant velocity $\mathbf{V}_{\mathbf{0}}$ making angle $\theta_{1}$ to the vector $\mathbf{H}_{\mathbf{0}}$ substantially exceeding root-mean-square velocity of turbulent mixing $|\mathbf{v}(\mathbf{r}, t)|<<\mathbf{V}_{\mathbf{0}}$. Let small amplitude $E_{0}$ monochromatic inertial low-frequency MHD wave with the frequency $\omega_{0}$ and the wave vector $\mathbf{k}_{\mathbf{0}}$ is generated in the plane $z=0$ making angle $\theta$ to the vector $\mathbf{H}_{\mathbf{0}}$. Electron density fluctuations surpass freezing-in magnetic field fluctuations and the conditions are fulfilled: $\left(H_{1} / H_{0}\right)<<\left(\left|N_{1}\right| / N_{0}\right)<<1$.

In the case of low-pressure plasma neglecting thermal velocities and ions motion under the action of the wave field, 
if electron density fluctuations are smooth and plasma nonstationarity is caused by turbulent motion, applying geometrical optics method [7] the following conditions are fulfilled:

$$
k_{0} l>>1, \frac{\omega_{0} l}{V_{0}}>>1
$$

where $l$ is characteristic linear scale of electron density irregularities. In this case we will neglect transformation of different waves and consider one of the most interesting case when collision frequency between plasma particles is small with respect to the frequency of an incident wave.

In the absence of temperature stratification, dissipation and compression only internal waves are generated in the ionospheric F-region under the action of geomagnetic field [8, 9]. New Alfven-type short-wave $\left(\lambda \leq 10^{3} \mathrm{~km}\right)$ and low-frequency inertial wave generating due to the Ampere electromagnetic force has been discovered in the ionospheric F region $[10,11]$.

In this paper statistical characteristics of scattered new inertial MHD wave in the ionospheric F-region are considered for the first time. Several specific features are arising at MHD wave propagation in the ionospheric plasma with spatial-temporal chaotically varying parameters, particularly energy exchange between the wave and nonstationary medium.

\section{Formulation of the Problem}

In the $\mathrm{F}$ region where the Hall effect is absent and $H_{0}=$ const, the linearized system of Helmholtz-Freedman equations for internal waves has the following form $[10,11]$ :

$$
\begin{gathered}
\frac{\partial \operatorname{rot} \mathbf{V}}{\partial t}=\operatorname{rot}\left[\mathbf{u} \cdot 2 \mathbf{\Omega}_{\mathbf{H}}\right], \frac{\partial \operatorname{rot} \mathbf{u}}{\partial t}=\operatorname{rot}\left[\mathbf{V} \cdot 2 \mathbf{\Omega}_{\mathbf{i}}\right], \\
\operatorname{div} \mathbf{V}=0, \operatorname{div} \mathbf{u}=0 .
\end{gathered}
$$

The condition $\operatorname{div} \mathbf{V}=0$ means that considered below perturbations are transverse waves; $\mathbf{V}$ is perturbation of the neutral particles velocity, $\mathbf{u}$ is vector potential having velocity dimension. Characteristic frequencies of the internal waves caused by geomagnetic field are:

$$
\begin{gathered}
2 \boldsymbol{\Omega}_{\mathbf{H}}=\frac{c k^{2}}{4 \pi e N} \mathbf{H}_{\mathbf{0}}=2 \Omega_{H} \boldsymbol{\tau}, \\
2 \boldsymbol{\Omega}_{\mathbf{i}}=\zeta \frac{e}{M c} \mathbf{H}_{\mathbf{0}}=2 \Omega_{i} \boldsymbol{\tau},
\end{gathered}
$$

where: $\boldsymbol{\tau}=\frac{\mathbf{H}_{\mathbf{0}}}{H_{0}}$ is a unit vector, $\zeta=\frac{N}{N+N_{n}} \approx \frac{N}{N_{n}}$ is the iconicity of ionospheric medium varying in $10^{-9}$ up to $10^{-3}$ in a height interval $80-600 \mathrm{~km}, M$ is the mass of an ion or molecule, $N_{n}$ is the concentration of neutral particles, $e$ and
$N$ are charge and concentration of electrons. Since the dynamic equations of system (2) are linear and sources and dissipation of energy are not taken into account, the energy of inertial waves cannot change in time and hence the wave frequency $\omega_{0}$ should be real.

One new branch of the Alfven-type low-frequency MHD wave has been revealed in the ionospheric F-region $[10,11]$ :

$$
\omega= \pm \omega_{a} \cos \theta
$$

where: $\omega_{a}=k V_{a}, \quad V_{a}=\frac{H_{0}}{\sqrt{4 \pi \rho}}=\sqrt{\zeta} \frac{H_{0}}{\sqrt{4 \pi M N}}, \quad \theta$ is angle between vectors $\mathbf{k}$ and $\mathbf{H}_{\mathbf{0}}$. Upper sign corresponds to the fast MHD wave, lower sign to the slow MHD wave. The density of neutral particles $\rho$ is important for the frequency $\omega_{a}$. The phase and group velocities of this wave are:

$$
V_{p h}=\frac{\omega}{k}=\frac{\omega_{a}}{k} \cos \theta, \quad V_{g r}=\frac{\partial \omega}{\partial \mathbf{k}}=\frac{\omega_{a}}{k}\{\boldsymbol{\tau}-\mathbf{n} \cos \theta\}
$$

The group velocity of this wave (velocity of propagation) is normal to wave vector $\mathbf{k}$; its absolute value and projection in direction $\mathbf{H}_{\mathbf{0}}$ would be written as:

$$
\left|V_{g r}\right|=\frac{\omega_{a}}{k} \sin \theta,\left(\mathbf{V}_{\mathbf{g r}} \cdot \boldsymbol{\tau}\right)=\frac{\omega_{a}}{k} \sin ^{2} \theta=\left|V_{g r}\right| \sin \theta
$$

From equations (5) and (6) follow, that when the group velocity has a component directed upward, the phase velocity has a component directed downward and vice versa $[10,11]$. Short inertial waves in the ionosphere belong to the class of very low-frequency (VLF) electromagnetic waves.

From equation (5) also follows that the information transfer to the Earth by the slow MHD waves by means of geomagnetic lines of force $H_{0}=$ const would not be complete, because a part of the information is lost owing to the second term in (5). From equation (4) follows that numerical values of the frequency of internal waves depend strongly on angle $\theta$. They reach the maximum at $\theta=0$ and disappear at $\theta=90^{\circ}$.

\section{Statistical Characteristics of Scattered Internal Wave in the Ionospheric F Region}

If the mean velocity $\mathbf{V}_{\mathbf{0}}$ of turbulent plasma flow makes angle $\theta_{1}$ to the external magnetic field $\mathbf{H}_{\mathbf{0}}$ and the wave vector of internal wave is located in the principle yoz -plane, the dispersion equation (4) can be rewritten as: $\omega-\left(\mathbf{k} \mathbf{V}_{\mathbf{0}}\right)= \pm \omega_{a} \cos \theta$. Using standard procedure [7] introducing the instant frequency $\omega(\mathbf{r}, t)=\partial \varphi / \partial t$ and wave vector $\mathbf{k}(\mathbf{r}, t)=|k| \mathbf{s}=-\nabla \varphi$ we obtain the eikonal equation for internal wave in the turbulent plasma flow. Phase we submit as: $\varphi(\mathbf{r}, t)=\varphi_{0}(\mathbf{r}, t)+\varphi_{1}(\mathbf{r}, t)$, where first is a regular term, the second one is stochastic term caused by electron 
density fluctuations, $\varphi_{1}<<\varphi_{0}$ and is random function of the spatial coordinates and time. Taking into account geometry of the task we obtain stochastic differential equation for the phase fluctuation:

$$
\frac{\partial \varphi_{1}}{\partial t}+A \frac{\partial \varphi_{1}}{\partial y}+B \frac{\partial \varphi_{1}}{\partial z}=\mp \frac{k_{0} V_{a 0} \cos \theta}{2} \tilde{n}_{1}
$$

where: $\quad A=V_{0} \sin \theta_{1} \mp \frac{\omega_{a 0}}{k_{0}} \sin \theta \cos \theta \quad, \quad \tilde{n}_{1}=N_{1} / N_{0}$, $B=V_{0} \cos \theta_{1} \mp \frac{\omega_{a 0}}{k_{0}} \cos ^{2} \theta \quad, \quad V_{a 0}=\sqrt{\zeta} H_{0} / \sqrt{4 \pi M N_{0}}$.

Using the method of characteristics in the region $z>0$ we obtain:

$$
\varphi_{1}(\mathbf{r}, t)=\mp \frac{\omega_{a 0} \cos \theta}{2 B} \int_{0}^{L_{z}} d z^{\prime} \tilde{n}_{1}\left(x, y^{\prime}, z^{\prime}, t^{\prime}\right)
$$

where: $y^{\prime}=y-A\left(z-z^{\prime}\right) / B, t^{\prime}=t-\left(z-z^{\prime}\right) / B$.

If electron density fluctuations are statistically homogeneous and stationary for arbitrary correlation function $W_{N}(\boldsymbol{\rho}, \tau)$ the variance of the phase fluctuation is:

$$
<\varphi_{1}^{2}>=\left(\frac{\omega_{a 0} \cos \theta}{2 B}\right)^{2} L_{z} \int_{-\infty}^{\infty} d \rho_{z} W_{N}\left(0, \rho_{y}, \rho_{z}, \tau\right),
$$

here: $\quad \rho_{y}=(A / B) \rho_{z}, \quad \tau=\rho_{z} / B ; \quad L_{z} \quad$ is a distance traversed by wave in the ionospheric plasma along the external magnetic field. Knowledge of this function allows to estimate dumping of the mean field of scattered wave using the well-known formula [12-14]:

$$
<E>=E_{0} \exp \left(-\frac{1}{2}<\varphi_{1}^{2}>\right) \text {. }
$$

One of the most important statistical characteristic in nonstationary media is the variance of instant frequency $\left.<\omega_{1}^{2}\right\rangle$ characterizing broadening of the temporal spectrum easily measuring by experiment:

$$
\begin{gathered}
<\omega_{1}^{2}>=2 \pi\left(\frac{\omega_{a 0} \cos \theta}{2 B}\right)^{2} L_{z} B \int_{-\infty}^{\infty} d \mathbf{k} \int_{-\infty}^{\infty} d \omega \omega^{2} \\
\cdot W_{N}\left(\mathbf{k}, A k_{y}+B k_{z}\right) .
\end{gathered}
$$

The degree of curvature of a constant phase surface in the turbulent plasma is characterized by fluctuations of a unite vector of the wave normal $\mathbf{s}$ :

$$
<s_{1 \perp}^{2}>=\frac{1}{k_{0}^{2}}<\left(\frac{\partial \varphi_{1}}{\partial x}\right)^{2}>=\left(\frac{\omega_{a 0} \cos \theta}{2 B}\right)^{2} L_{z}
$$

$$
\int_{-\infty}^{\infty} d \rho_{z}\left(\frac{\partial^{2}}{\partial \rho_{x}^{2}}+\frac{\partial^{2}}{\partial \rho_{y}^{2}}\right) W_{N}\left(0, \rho_{y}, \rho_{z}, \tau\right)
$$

In the geometrical optics approximation neglecting dissipation processes, transport equation for the wave amplitude $E$ or the logarithmic relative amplitude $\chi=\ln \left(E / E_{0}\right)$ can be obtained from the differential equation [7]:

$$
\frac{\partial}{\partial t}\left(\eta E^{2}\right)+\operatorname{div}\left(V_{g r} \eta E^{2}\right)=-\frac{\partial \varepsilon}{\partial t} E^{2}
$$

where: $\varepsilon=\frac{c^{2}}{V_{a}^{2}} \frac{\left(\omega-\mathbf{k} \mathbf{V}_{\mathbf{0}}\right)^{2}}{\omega^{2}}$ is the dielectric permittivity, $\eta=\frac{1}{\omega} \frac{\partial}{\partial \omega}\left(\omega^{2} \varepsilon\right)$ is the coefficient connecting energy density and $E^{2}, \boldsymbol{\Phi}=\mathbf{V}_{\mathbf{g r}} \eta E^{2}$ is the energy flux density.

Substituting in equation (13) the expressions:

$$
\begin{gathered}
\frac{\partial}{\partial t}\left(\eta E^{2}\right)=2 \frac{c^{2}}{V_{a 0}^{2}} E_{0}^{2} \eta_{0}\left(2 \frac{\partial \chi_{1}}{\partial t}-\frac{\partial \tilde{n}_{1}}{\partial t}\right) \\
\frac{\partial \varepsilon}{\partial t} E^{2}=2 \frac{c^{2}}{V_{a 0}^{2}} E_{0}^{2} \varepsilon_{0} \frac{\partial \tilde{n}_{1}}{\partial t}
\end{gathered}
$$

we obtain stochastic differential equation of the logarithmic relative amplitude $\chi_{1}$ the solution of which is:

$$
\chi_{1}(\mathbf{r}, t)=\frac{1}{V_{20 z}} \int_{0}^{L_{z}} d z^{\prime}\left(D_{1} \frac{\partial \tilde{n}_{1}}{\partial t^{\prime}}+D_{2} \frac{\partial \tilde{n}_{1}}{\partial y^{\prime}}\right)
$$

where: $\quad V_{20 z}=V_{0} \cos \theta_{1} \pm V_{a 0} \sin ^{2} \theta \quad, \quad D_{1}=\frac{1}{2}\left(1+\frac{\Delta_{0}}{4}\right)$, $\Delta_{0}=1-g_{0} \cos \left(\theta-\theta_{1}\right), \quad g_{0}=k_{0} V_{0} / \omega_{0}, \omega_{0}$ is determined from the dispersion equation of the internal wave, $D_{2}=\frac{1}{2}\left(V_{0} \sin \theta_{1} \mp \frac{3}{2} V_{a 0} \sin \theta \cos \theta\right)$. Characteristics are: $y^{\prime}=y-\frac{A}{V_{20 z}}\left(z-z^{\prime}\right) \quad, \quad t^{\prime}=t-\frac{1}{V_{20 z}}\left(z-z^{\prime}\right) \quad . \quad$ When fluctuations of the field are small and the forward scattering approximation is valid, the usually observed scintillation index $S_{4}$ is proportional to $S_{4}^{2} \approx 4<\chi_{1}^{2}>[15]$.

The next important problem of wave propagation in nonstationary medium is the energy exchange between the inertial wave and turbulent ionospheric plasma. The mean energy flux density (EFDE) of internal wave along the $z$ direction is the sum of two terms in zero and second approximations:

$$
<\Phi_{z}>=\Phi_{z 0}+<\Phi_{z}>_{2}=2 \frac{c^{2}}{V_{a 0}^{2}} E_{0}^{2} \Delta_{0} V_{20 z}\left[1-\frac{\Delta_{0} L_{z}}{V_{20 z}^{2}} .\right.
$$




$$
\left.\int_{-\infty}^{\infty} d \rho_{z}\left(D_{1} \frac{\partial^{2}}{\partial \tau^{2}}+D_{2} \frac{\partial^{2}}{\partial \rho_{y} \partial \tau}\right) W_{N}\left(0, \rho_{y}, \rho_{z}, \tau\right)\right]
$$

Growth of the energy flux along the $z$-axis means the energy transfer from the medium to the wave and energy decrease vice versa - from wave to the medium. These formulae are valid for near $\left(L / k_{0} l^{2}\right)<<1$ and far $\left(L / k_{0} l^{2}\right)>>1$ zones from a plasma slab boundary.

\section{Numerical Calculations}

Experimental observations using orbital satellite beacons and the EISCAT incoherent scatter radar show [15] that under quite geomagnetic conditions for a cross-field irregularity scale $l_{\perp} \approx 1 \mathrm{~km}$ a plasma drift velocity was basica-

lly westwards and $V_{0}=0.3 \mathrm{~km} / \mathrm{s}$. The field-aligned scale of irregularities was of the order of $l_{\|} \approx 30 \mathrm{~km}$ with the plasma drift velocity $(1-2) \mathrm{km} / \mathrm{s}$. Since the inertial waves propagate in the F-region of the ionosphere, at $\theta=0$ and $\lambda=10^{2} \mathrm{~km}$ the frequencies of this wave in the altitudes $h=150-300 \mathrm{~km}$ are in the interval $1.5 \cdot 10^{-2}-1.5 \cdot 10^{-1}$ [10]. Other experimental data of the slow MHD waves measured by ground-based radar systems are presented in [16]. It was shown that slow MHD waves perturbations can be propagate over distances $\sim 1000 \mathrm{~km}$ at $\omega \leq 10^{-2} \mathrm{sec}^{-1}$ (period $T \geq 10$ minute), while on global distances at $\omega \leq 10^{-3} \mathrm{sec}^{-1}$ (period $T \geq 100$ minute). Phase velocity of these waves are in the interval $6 \div 10 \mathrm{~km} / \mathrm{sec}$ and less.

The irregularity model is described by $3 \mathrm{D}$ correlation function of electron density fluctuations. Numerical calculations are carried out for both Gaussian and power-law spectra for elongated ionospheric irregularities. The spectral density function which best describes the irregularities in a randomly inhomogeneous magnetized plasma depends on the particular case. Most widely used the Gaussian spectral function in the Gaussian, which has certain mathematical advantages. However, power-law spectral densities have been receiving more attention as being physically more realistic. Knowledge of the power spectrum of ionospheric refractive index fluctuations can lead to an understanding of the physical processes that characterize the region of the ionosphere under study.

Observations by "Sura" heating facility experiment show [17] that artificial ionospheric irregularities are stretched along the geomagnetic field. It was established that the transversal scale $l_{\perp}$ of elongated irregularities varies in the range of $100-500 \mathrm{~m}$; the magnitudes of the drift velocity were within the limits $65-270 \mathrm{~m} / \mathrm{s}$ (the typical velocities of ionospheric motions $V_{0} \approx 60-100 \mathrm{~m} / \mathrm{s}$ ). Velocity $100 \mathrm{~m} / \mathrm{s}$ caused by the steady drifting with the horizontal wind of scattering irregularities embedded in the ionosphere will be used in numerical calculations.

Data obtained from spaced receiver measurements made at Kingston, Jamaica (during the periods August 1967- January
1969 and June 1970-September 1970) show that the irregularities responsible for fluctuations of MHD waves parameters and causing the scintillation of signals from the moving earth satellites (BE-B and $\mathrm{BE}-\mathrm{C}$ ) are between heights of 153 and $617 \mathrm{~km}$ closely aligned along the magnetic field lines in the $F$-region [18]. Orientation of the irregularities in the ionosphere has been measured with respect to the geographic north observing a diffraction pattern of the satellite signals $(41 \mathrm{MHz})$ on the ground. The dip angle of the irregularities with respect to the field lines was within $16^{0}$.

In the geometrical optics approximation forward scattering assumption is valid: $\sigma_{N}^{2} k_{0} L_{z} \ll 1<<k_{0} l, \quad \sigma_{N}^{2}$ is the variance. If the single scattering condition is also fulfilled $\sigma_{N}^{2} k_{0}^{2} l L<<1$ a medium is characterized by the Gaussian irregularity spectrum [15].

Numerical calculations are carrying out for the anisotropic Gaussian correlation function of electron density fluctuations [19]:

$$
\begin{aligned}
\mathrm{W}_{N}(\mathbf{k}, \omega) & =\sigma_{N}^{2} \frac{l_{\perp}^{2} l_{\|}}{8 \pi^{3 / 2}} \frac{T}{2 \sqrt{\pi}} \exp \left(-\frac{k_{x}^{2} l_{\perp}^{2}}{4}-p_{1} \frac{k_{y}^{2} l_{\|}^{2}}{4}-\right. \\
-p_{2} & \left.\frac{k_{z}^{2} l_{\|}^{2}}{4}-p_{3} k_{y} k_{z} l_{\|}^{2}\right) \exp \left(-\frac{\omega^{2} T^{2}}{4}\right)
\end{aligned}
$$

where: $p_{3}=\frac{\chi^{2}-1}{2 \chi^{2}} \sin \gamma_{0} \cos \gamma_{0}, \quad p_{1}=\frac{1}{\chi^{2} \cos ^{2} \gamma_{0}+\sin ^{2} \gamma_{0}}$

$$
\cdot\left[1+\frac{\left(\chi^{2}-1\right)^{2}}{\chi^{2}} \sin ^{2} \gamma_{0} \cos ^{2} \gamma_{0}\right], p_{2}=\frac{\sin ^{2} \gamma_{0}+\cos ^{2} \gamma_{0}}{\chi^{2}},
$$

$\chi=l_{\|} / l_{\perp}$ is the anisotropy factor of field-aligned irregularities defined as a ratio of longitudinal and transversal characteristic liner scales of electron density irregularities, $\gamma_{0}$ is the slop angle of elongated plasma irregularities with respect to the geomagnetic field, $T$ is the characteristic temporal scale of ionospheric plasma irregularities; $1 / T$ is the own frequency of irregularities pulsations. The average shape of electron density irregularities has the form of elongate ellipsoid of rotation. The rotation axis is located in the plane of geomagnetic meridian. Anisotropy of the shape of irregularities is connected with the difference of diffusion coefficients in the field align and field perpendicular directions.

Measurements of satellite's signal parameters passing through ionospheric layer and measurements aboard of satellite show that irregularities in $F$-region of the ionosphere have power-law spectrum with different spatial scales. The 3D power spectrum of electron density fluctuations adequately explaining observing plasma irregularities having linear scale larger than $800 \mathrm{~m}$ has the power-law index $2<p<6$ [19]: 


$$
\begin{gathered}
W_{N}(\mathbf{k})=\frac{\sigma_{N}^{2}}{2 \pi^{2}} \frac{\Gamma\left(\frac{p}{2}\right) \Gamma\left(\frac{5-p}{2}\right)}{\Gamma\left(\frac{3}{2}\right)} \cdot \sin \left[\frac{(p-3) \pi}{2}\right] . \\
\cdot \frac{l_{\perp}^{2} l_{\|}}{\left[1+l_{\perp}^{2}\left(k_{\perp}^{2}+\chi^{2} k_{\|}^{2}\right)\right]^{p / 2}}
\end{gathered}
$$

where: $\Gamma(x)$ is the gamma function.

Substituting (17) into (9) we obtain:

$$
<\varphi_{1}^{2}>=\frac{\sqrt{\pi}}{4} \sigma_{N}^{2} \frac{\omega_{a 0}^{2} L_{z} T}{\sqrt{q_{3}} B \chi^{2}} \cos ^{2} \theta\left(q_{4}-\frac{q_{5}^{2}}{q_{3}}\right)^{-1 / 2}
$$

where: $\quad q_{3}=p_{1}+\left(T^{2} / l_{\|}^{2}\right) A^{2} \quad, \quad q_{4}=p_{2}+\left(T^{2} / l_{\|}^{2}\right) B^{2}$, $q_{5}=p_{3}-\left(T^{2} / 2 l_{\|}^{2}\right) A B$.

For spectral function (17) variance of an instant frequency (11) and the degree of a curvature of a constant phase surface (12) along the direction of geomagnetic field of lines $(z-$ direction) yield:

$$
\begin{aligned}
& \frac{<\omega_{1}^{2}>_{z}}{\omega_{0}^{2}}=\frac{\sqrt{\pi}}{2} \sigma_{N}^{2} \frac{\omega_{a 0}^{2}}{\omega_{0}^{2}} \cdot \frac{L_{z}}{l_{\|}} \cdot \frac{T \cos ^{2} \theta}{\chi l_{\|} B} \\
& {\left[\frac{A^{2}}{\sqrt{q_{4}}}\left(q_{3}-4 \frac{q_{5}^{2}}{q_{4}}\right)^{-3 / 2}+\frac{B}{\sqrt{q_{3}}}\left(B+4 A \frac{q_{5}}{q_{3}}\right) .\right.} \\
& \left.\left(q_{4}-4 \frac{q_{5}^{2}}{q_{3}}\right)^{-3 / 2}\right] \\
& \frac{<k_{1 \perp}^{2}>_{z}}{k_{0}^{2}}=\frac{\sqrt{\pi}}{2} \sigma_{N}^{2} \frac{L_{z}}{k_{0} l_{\|}^{2}} \cdot \frac{\omega_{a 0}^{2} T}{\chi B k_{0}} \cos ^{2} \theta \\
& {\left[\frac{\chi^{2}}{\sqrt{q_{3}}}\left(q_{4}-4 \frac{q_{5}^{2}}{q_{3}}\right)^{-1 / 2}+\frac{1}{\sqrt{q_{4}}}\left(q_{3}-4 \frac{q_{5}^{2}}{q_{4}}\right)^{-3 / 2}\right] .}
\end{aligned}
$$

Energy exchange between inertial wave propagating in the ionospheric $\mathrm{F}$ region and the regular turbulent plasma flow depends on the following parameters: angles $\theta$ and $\theta_{1}$; characteristic spatial scale of plasma irregularities $l_{\|}$and the frequency of theirs temporal pulsation $1 / T$; velocities $V_{0}$ and $V_{a 0}$; distance traveling by inertial wave in the turbulent plasma $L_{y, z} / l_{\|}$and $L_{y, z} /\left(k_{0} l_{\|}^{2}\right)<<1$ (this condition should be fulfilled as in the geometrical optics approximation diffraction effects are neglect [12]-[14], however despite of this the obtain results are good approach to the reality (especially phase)); frequencies $\omega_{0}$ and $\omega_{a 0}$; wavenumber $k_{0}$.
Substituting (17) in equation (16) we obtain:

$$
\begin{aligned}
&<\Phi_{z}>=2 \frac{c^{2}}{V_{a 0}^{2}} E_{0}^{2} \Delta_{0} V_{20 z}\left\{1-2 \sqrt{\pi} \sigma_{N}^{2} \cdot \frac{\Delta_{0} L_{z}}{V_{20 z}^{2}} .\right. \\
& \cdot \frac{B T}{l_{\|}^{2} \chi}\left[\frac{q_{0}}{\sqrt{q_{4}}}\left(q_{3}-4 \frac{q_{5}^{2}}{q_{4}}\right)^{-3 / 2}+\right. \\
&\left.\left.+\frac{1}{\sqrt{q_{3}}}\left(-q_{1}+2 \frac{q_{2} q_{5}}{q_{3}}\right) \cdot\left(q_{4}-4 \frac{q_{5}^{2}}{q_{3}}\right)^{-3 / 2}\right]\right\}, \\
&<\Phi_{y}>=2 \frac{c^{2}}{V_{a 0}^{2}} E_{0}^{2} \Delta_{0} A\left\{1-2 \sqrt{\pi} \sigma_{N}^{2} \cdot \frac{\Delta_{0} L_{y}}{A} .\right.
\end{aligned}
$$

$$
\begin{gathered}
\cdot \frac{T}{l_{\|}^{2} \chi}\left[-\frac{q_{6}}{\sqrt{q_{4}}}\left(q_{3}-4 \frac{q_{5}^{2}}{q_{4}}\right)^{-3 / 2}+\right. \\
\left.\left.+\frac{1}{\sqrt{q_{3}}}\left(q_{7}+2 \frac{q_{5} q_{8}}{q_{3}}\right) \cdot\left(q_{4}-4 \frac{q_{5}^{2}}{q_{3}}\right)^{-3 / 2}\right]\right\},
\end{gathered}
$$

where: $q_{0}=A\left(D_{2}-D_{1} A\right), q_{1}=D_{1} B^{2}, q_{6}=D_{1} A^{2}$,

$$
\begin{gathered}
q_{2}=B\left(D_{2}-2 D_{1} A\right), q_{7}=B\left(D_{3}-B D_{1}\right), \\
q_{8}=A\left(D_{3}-2 D_{1} B\right), \therefore D_{3}=\frac{1}{2}\left(V_{0} \cos \theta_{1} \pm \frac{1}{2} V_{a 0} \sin ^{2} \theta\right) .
\end{gathered}
$$

If the second term in brace is negative this means that turbulent plasma transfer its energy to the inertial wave increasing its amplitude and intensity propagating along the geomagnetic lines of force; if second term is negative internal wave losses energy transferring it to the plasma flow and its intensity decreases.

Applying the spectral function of the spatial-temporal power spectrum of electron density fluctuation in the "frozen" turbulence $V_{N}(\boldsymbol{\kappa}, \Omega)=F_{N}(\boldsymbol{\kappa}) \delta\left(\Omega-\mathbf{\kappa} \mathbf{V}_{\mathbf{0}}\right) \quad$ [12-14] from equation (9) for the variance of the phase fluctuations in the principle plane we obtain:

$$
<\varphi_{1}^{2}>=\frac{\pi}{2} \frac{\omega_{a 0} k_{0} L_{z}}{B N_{0}^{2}} \int_{-\infty}^{\infty} d \kappa_{x} \int_{-\infty}^{\infty} d \kappa_{y} F_{N}\left(\kappa_{x}, \kappa_{y}, \kappa_{y} \cdot \operatorname{tg} \theta\right)
$$

In this case the mean energy flux density along the z-axis is:

$$
\begin{gathered}
<\Phi_{z}>=\pi L_{z} \frac{c^{2}}{V_{a 0}^{2}} \cdot \frac{\omega_{*}^{2}}{\omega_{0}^{2}} \cdot \frac{V_{0}\left(\Lambda_{1}-Q_{2}^{2} \Lambda_{2}-Q_{2} \Lambda_{3}\right)}{V_{0} \sin \theta_{1}+V_{a 0} \sin \theta \cos \theta} \\
\int_{-\infty}^{\infty} d \kappa_{x} \int_{-\infty}^{\infty} d \kappa_{z} \kappa_{z}^{2} F_{N}\left(\kappa_{x},-Q_{2} \kappa_{z}, \kappa_{z}\right),
\end{gathered}
$$




$$
\begin{gathered}
\Lambda_{1}=\cos \theta_{1}\left(3 V_{a 0} \sin ^{2} \theta-b V_{0} \cos \theta_{1}\right), b=2-\left(\omega_{*} / \omega_{0}\right), \\
\Lambda_{2}=\sin \theta_{1}\left(3 V_{a 0} \sin \theta \cos \theta+b V_{0} \sin \theta_{1}\right), \\
\Lambda_{3}=3 V_{a 0} \sin \theta\left(\sin \theta \sin \theta_{1}-\cos \theta \cos \theta_{1}\right)- \\
-2 b V_{0} \sin \theta_{1} \cos \theta_{1}, \omega_{*}=\omega_{0}-\left(\mathbf{k}_{0} \mathbf{V}_{\mathbf{0}}\right) .
\end{gathered}
$$

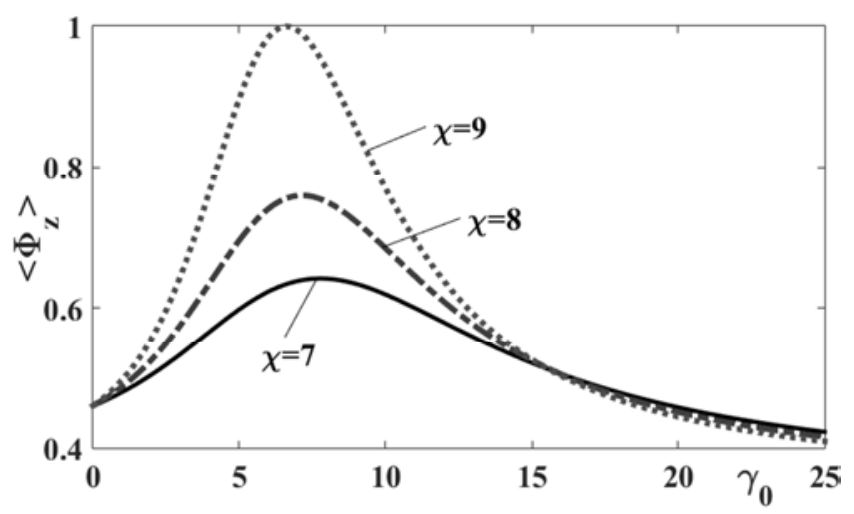

Figure 1. The mean EFDE along $z$ direction versus slope angle $\gamma_{0}$ for different anisotropy factor $\chi$.

Figure 1 depicts normalized mean EFDE of the internal wave along the directions to the external magnetic field versus slope angle of elongated plasma irregularities $\gamma_{0}$ for different anisotropy factor $\chi$. Velocity of the turbulent plasma flow is directed along lines of force of geomagnetic field $\left(\theta_{1}=0^{0}\right)$ and the angle $\theta=5^{0}$. Longitudinal characteristic spatial scale of large-scale ionospheric plasma irregularities is $l_{\|}=10 \mathrm{~km}$. Numerical calculations show that turbulent ionospheric plasma flow transfer energy to the internal wave increasing its intensity in proportion to the parameter $\chi$ : traveling distance

$10^{3} \mathrm{~km}$ in the ionospheric $\mathrm{F}$ region maximum of the curves is displaced to the direction of an external magnetic field. Particularly, at $T=10 \mathrm{sec}$ varying anisotropy factor in the interval $2 \leq \chi \leq 9$ maximum of both functions $\left\langle\Phi_{z}\right\rangle_{\max }$ and $\left\langle\Phi_{y}\right\rangle_{\max }$ (normal to the external magnetic field) shifts from $\gamma_{0}=17^{0}$ to the $\gamma_{0}=8^{0}$. Increasing characteristic temporal scale of electron density irregularities up to $T=400 \mathrm{sec}$ maximum of a curve $\left\langle\Phi_{z}\right\rangle_{\max }$ is displaced in the interval $5^{0} \leq \gamma_{0} \leq 4^{0}$. Hence, internal wave propagating in the ionospheric $\mathrm{F}$ region with large scale electron density irregularities near the turbulent plasma flow pumps energy growing its energy flux; increasing anisotropy factor of elongated plasma irregularities maximum of EFDE is displaced to the direction of lines of force of geomagnetic field; decreasing own frequency $(1 / T)$ of the turbulent ionospheric plasma irregularities interval of slop angle of elongated plasma irregularities having substantial influence on the energy flux density substantially narrows.

Figure 2 illustrates normalized mean EFDE as a function of anisotropy factor for different inclination angle of elongated electron density irregularities with respect to the external magnetic field. Numerical analyses show that the mean EFDE of the internal wave is increased inversely proportion to the angle $\gamma_{0}$ due to energy exchange with the plasma flow. MHD wave travelling distance $1000 \mathrm{~km}$ near to the lines of force of geomagnetic field pumps energy from the turbulent plasma flow increasing its intensity along and normal directions to the external magnetic field. Varying slop angle in small interval $20^{0} \leq \gamma_{0} \leq 23^{0}$ broadening of both functions $<\Phi_{z}>$ and $\left.<\Phi_{y}\right\rangle$ decrea-ses on $62 \%$. So, increasing the angle of inclination of elongated plasma irregularities even in small interval the width of the EFDE substantially decreases.

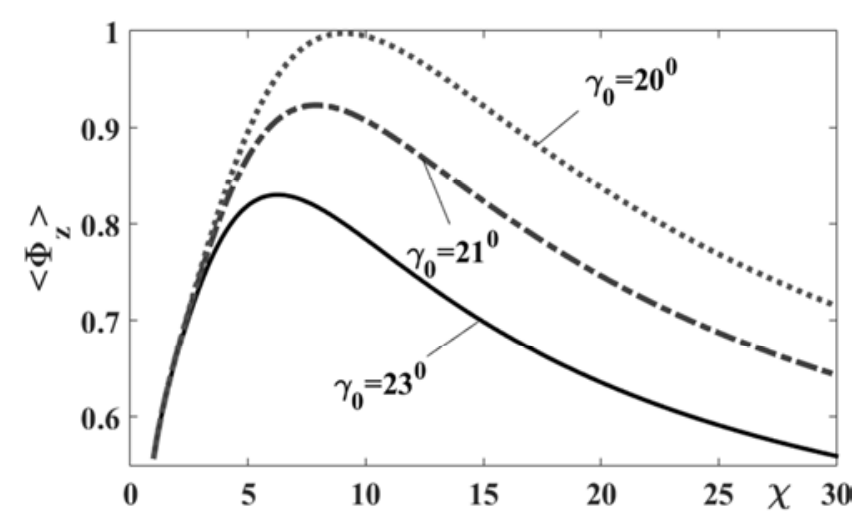

Figure 2. The mean EFDE along $z$ direction versus anisotropy factor $\chi$ for different inclination angle $\gamma_{0}$.

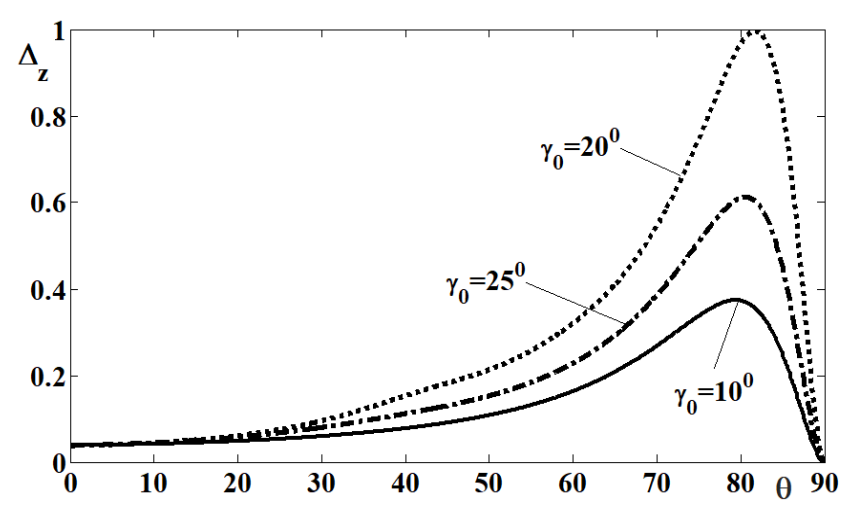

Figure 3. Variance of the instance frequency of scattered inertial wave versus angle of its propagation for different slope angle.

Figure 3 depicts normalized variance of the instance frequency $\left.\Delta_{z} \equiv<\omega_{1}^{2}\right\rangle_{z} / \omega_{0}^{2}$ determining the broadening of the temporal power spectrum of scattered inertial wave versus angle propagating in the ionospheric $F$ region for different slope angle of elongated plasma irregularities. Analyses show that maximum and shift of maximum of the spectrum depends on both the directions of plasma flow and internal wave propagation with respect to the external magnetic field. Numerical calculations are carried out for fixed parameters $\chi=2$ and $T=10 \mathrm{sec}$. Increasing angles 
$\gamma_{0}$ and $\theta_{1}$ spectrum narrows and its maximum displaces to the direction of big angle $\theta$. If the angle $\theta_{1}$ is in the interval $0^{0} \leq \theta_{1} \leq 70^{0}$ maximum of the temporal spectrum shifts to the right (to the big angles). Particularly, at $\gamma_{0}=10^{0}$ maximum displaced on $5^{0}$ degree, for $\gamma_{0}=20^{0}$ - on $8^{0}$, while at $\gamma_{0}=30^{0}-$ on $11^{0}$; if $\gamma_{0}$ is in the interval $0^{0} \leq \gamma_{0} \leq 30^{0}$ the curve narrows on $71 \%$ percent.

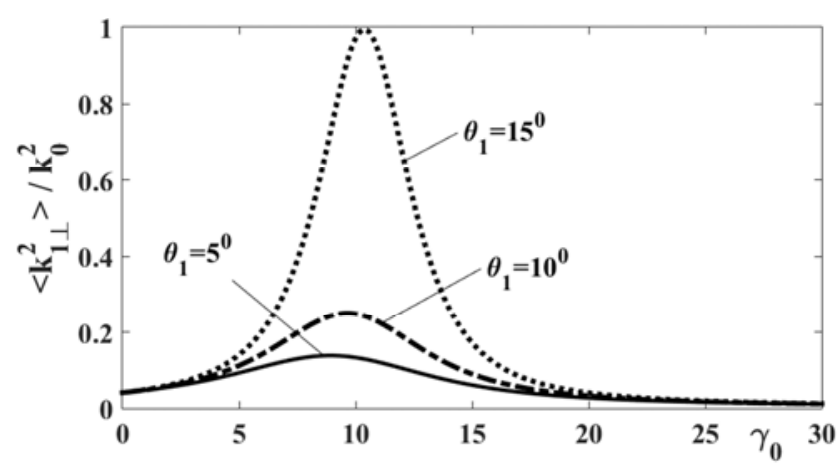

Figure 4. Degree of a curvature of a constant phase surface versus slope angle of elongated plasma irregularities.

Figure 4 illustrates degree of a curvature of a constant surface $\theta=5^{0}$ in the ionospheric $\mathrm{F}$ region. Velocity of a turbulent plasma flow is $V_{0}=150 \mathrm{~m} / \mathrm{sec}$ making angle $\theta_{1}=5^{0}, 10^{0}$ and $15^{0}$ to the external magnetic field; anisotropy factor of elongated plasma irregularities is $\chi=7$, $T=10$ sec. Numerical calculations show that internal wave passing distance $L_{z}=100 \mathrm{~m}$ in the turbulent plasma flow varying angle from $\theta_{1}=10^{0}$ up to $15^{0}$ the degree of a curvature increases four times. Maximum of a curve and its survature degree increases in proportion to the distance travelling by wave in the turbulent plasma.

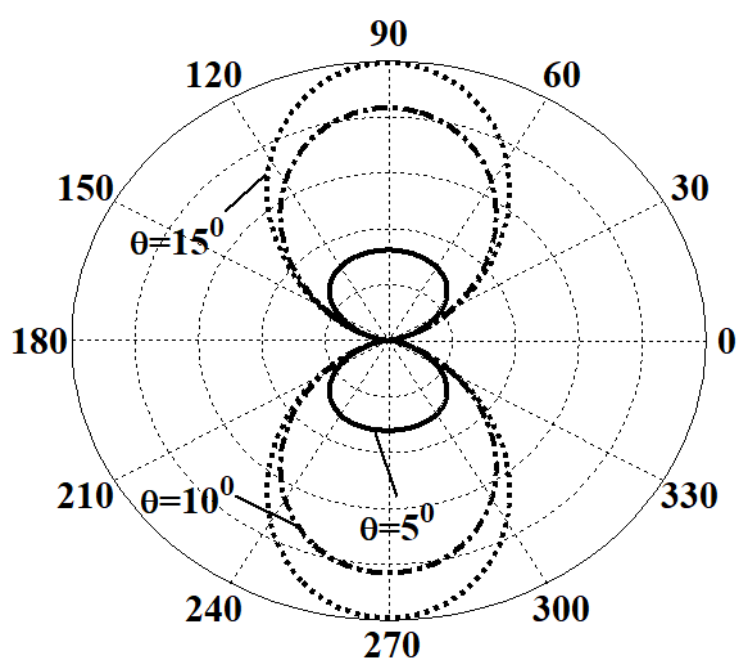

Figure 5. Phase portrait of $\left\langle\Phi_{z}\right\rangle$ in the polar coordinate system for frozen plasma irregularities varying angle $\theta$
Figure 5 represents phase portrait in the polar coordinate system for the mean EFDE of the internal wave along the $z$ direction in the case of frozen turbulence. Numerical calculations show that in isotropic case $(\chi=1)$ when the directions of plasma flow and external magnetic field coincide $\left(\theta_{1}=0^{0}\right)$ and the inertial wave propagates at angles $\theta=5^{0}, 10^{0}, 15^{0}$ with respect to the geomagnetic lines of forces the curves have dipole forms. Analyses show that change of the angle $\theta_{1}$ has not influence of the phase portrait, while lobes of scattered pattern become bigger in proportion to the angle $\theta$.

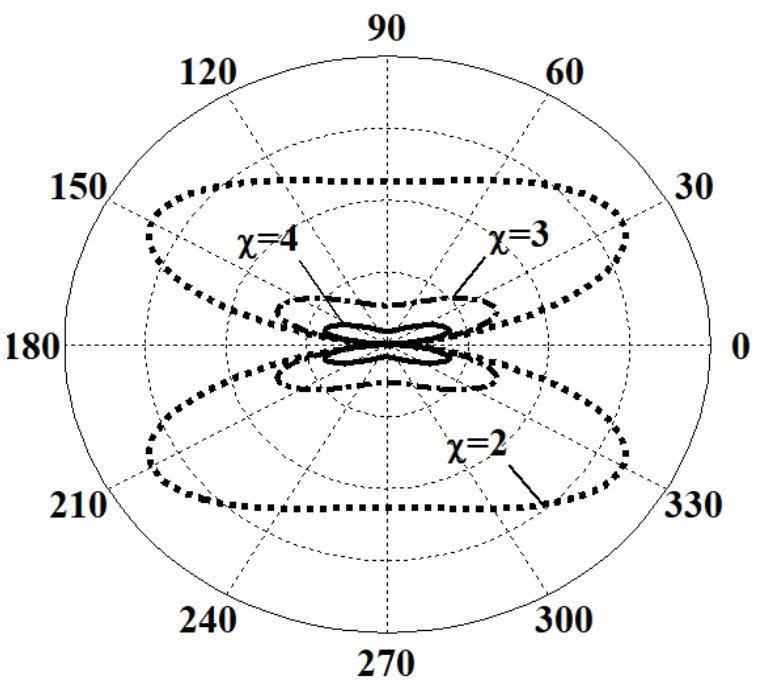

Figure 6. Phase portrait of $\left\langle\Phi_{z}\right\rangle$ in the polar coordinate system for frozen plasma irregularities varying anisotropy factor $\chi$.

Figure 6 depicts phase portrait of the mean EFDE in the frozen magnetized plasma if the inertial wave propagates along the geomagnetic field of lines $\left(\theta=0^{0}\right)$ at fixed angle $\theta_{1}=50^{\circ}$ varying anisotropy factor of elongated plasma irregularities $\chi=2,3,4$. Numerical calculations show that lobes of scattered pattern become flatter increasing angle $\theta$ while they become inflated in proportion to the angle $\theta_{1}$.

\section{Acknowledgements}

The work has been supported by the International Science and Technology Center (ISTC) under Grant \# G-2126 and Shota Rustaveli National Science Foundation under Grant \# FR/3/9-190/14.

\section{References}

[1] A. I. Akhiezer, I. A. Akhiezer, R. V. Polovin, A. G. Sitenko, and K. N. Stepanov, "Electrodynamics of Plasma," Moscow, Nauka, 1974 (in Russian).

[2] V. M. Sorokin, and G. V. Fedorovich, "Physics of Slow MHD Waves in the Ionospheric Plasma," Nauka, Moscow, 1982 (in Russian). 
[3] A. F. Aleksandrov, L. S. Bogdankevich, and A. A. Rukhadze, Electrodynamics of Plasma, Moscow, Higher Educational Institution, 1988 (in Russian).

[4] G. V. Jandieri, V. G. Gavrilenko, and A. A. Semerikov, "To the Theory of MHD Waves Propagation in the Turbulent Plasma Flow," Plasma Physics, vol. 11, \# 10, pp. 1193-1198, 1985 (in Russian).

[5] G. V. Jandieri, "To the Problem of MHD Wave Propagation in the Ionospheric E-region," Journal of Applied Science and Research, vol. 2, \# 2, pp. 1-13, 2014.

[6] G. V. Jandieri, O. Kharshiladze, Zh. M. Diasamidze, M. R. Diasamidze, "Magnetosonc Waves in the Turbulent Ionospheric Plasma," International Journal of Advancement in Earth and Environmental Science,” vol. 2, \# 1, pp. 51-62, 2014.

[7] Yu. A. Kravtsov, and Yu. I. Orlov, "Geometrical Optics of Inhomogeneous Media,” Moscow, Nauka, 1980 (in Russian).

[8] J. Pedlosky, "Geophysical Fluid Dynamics", (Springer, New York, 1982, Mir, Moscow, 1984).

[9] L. D. Landau, and I. M. Lifshits, "Fluid Mechanics," (Pergamon Press, Oxford, 1987; Nauka, Moscow, 1988).

[10] A. G. Khantadze, G. G. Lominadze, and G. V. Jandieri, "Internal Waves in the Ionosphere Related to the Earth's Geomagnetic Field," Doklady Earth Science, vol. 420, \# 4, pp. 672-675, 2008.

[11] A. G. Khantadze, and G. V. Jandieri, “General-planetary Character of Three-dimensional Planetary Waves Propagation in Lower and Upper Earth Atmosphere," J. of Atmospheric and Solar-Terrestrial Physics, vol. 71, pp. 45-48, 2009.

[12] A Ishimaru, "Wave Propagation and Scattering in Random Media, Vol. 2, Multiple Scattering, Turbulence, Rough
Surfaces and Remote Sensing," IEEE Press, Piscataway, New Jersey, USA, 1997.

[13] V. I. Tatarskii, "Wave Propagation in a Turbulent Medium," McGraw-Hill, New York, 1961.

[14] S. M. Rytov, Yu. A. Kravtsov, and V. I. Tatarskii, "Principles of Statistical Radiophysics. vol. 4. Waves Propagation Through Random Media. Berlin, New York, Springer, 1989.

[15] A. W. Wernik, and C. H. Liu, "Ionospheric Irregularities Causing Scintillation of GHz Frequency Radio Signals," J. of Atmospheric and Terrestrial Physics, vol. 36, pp. 871-879, 1974.

[16] V. P. Burmaka, L. S. Kostrov L. S., and L. F. Chernogor, "Statistics of Signals of HF Doppler Radar Probing the Bottomside Ionosphere Distributed by Rocket Launches and Solar Terminator," Radio Physics and Radio Astronomy, vol. 8, \# 2 pp. 143-162, 2003 (in Russian).

[17] N. V. Bakhmet'eva, V. N. Bubukina, Yu. A. Ignat'ev, G. S. Bochkarev, V. A. Eremenko, V. V. Kol'sov, I. V. Krasheninnikov and Yu. N. Cherkashin, "Investigation by Backscatter Radar Irregularities Produced in Ionospheric Plasma Heating Experiments," Journal of Atmospheric and Terrestrial Physics, vol. 59, \# 18, pp. 2257-2263, 1997.

[18] A. A., and G. S. Kent, "Determination of the Orientation of Ionospheric Irregularities Causing Scintillation of Signals From Earth Satellites," Journal of Atmospheric and Terrestrial Physics, vol. 34, pp. 1411-1414, 1972.

[19] G. V. Jandieri, A. Ishimaru, V. G. Jandieri, A. G. Khantadze, and Zh. M. "Model Computations of Angular Power Spectra for Anisotropic Absorptive Turbulent Magnetized Plasma," PIER, vol. 70, pp. 307-328, 2007. 